\title{
Reversible cerebral vasoconstriction syndrome (RCVS) in the context of aseptic meningitis in a twenty eight year-old postpartum female
}

\author{
Alyssa L. Arena*, Xiangkun Cao, Bryce J. Hamilton, Dipendra Chaudhary \\ Wake Forest School of Medicine, North Carolina, United States
}

Received: September 11, 2018

Accepted: January 28, 2019

Online Published: January 30, 2019

DOI: $10.5430 /$ crim.v6n1p16

URL: https://doi.org/10.5430/crim.v6n1p16

\begin{abstract}
Reversible cerebral vasoconstriction syndrome (RCVS) is an uncommon pathology that typically presents with a severe thunderclap headache in middle-aged postpartum females. Our patient was a twenty eight year-old Caucasian female seven months postpartum who presented with a ten day history of severe frontal headaches, myalgia, nausea, and anorexia without fevers. She then developed severe left hemiplegia, dysarthria, and paresthesias. Initial cerebrospinal fluid was significant for lymphocytepredominant pleocytosis, and acyclovir, vancomycin, and doxycycline were started for empiric meningitis. Cultures and markers were pan-negative for viral, bacterial, fungal, and autoimmune etiology. A computed tomography head scan was suggestive of distal right internal carotid artery atherosclerosis and ectasia, highly unusual for a woman of this age with no cardiovascular risk factors. Her hospital course was characterized by insomnia, delirium, worsening overnight headaches, and unusual bradycardic episodes. A computed tomography angiogram confirmed right internal carotid artery and middle cerebral artery M1 and M2 segment narrowing, suggestive of RCVS. A follow-up cerebral arteriogram highlighted right middle cerebral artery M1 segment tapering, confirming this diagnosis. We suspect an additional diagnosis of aseptic meningitis, due to her cerebrospinal fluid findings, which likely exacerbated her clinical presentation. Her refractory headaches eventually improved spontaneously. This presentation of reversible cerebral vasoconstriction syndrome in the context of a late post-partum onset and aseptic meningitis is the first such case to be documented, and we hope to shed some light on this unusual and uncommon disease, as well as some avenues for future research.
\end{abstract}

Key Words: Aseptic, Meningitis, Vasoconstriction, Thunderclap, Postpartum

\section{INTRODUCTION}

Reversible cerebral vasoconstriction syndrome (RCVS) is an uncommon pathology that typically presents in middle-aged females with "thunderclap" headaches due to diffuse cerebral artery constriction. ${ }^{[1]}$ Triggers include orgasm, voiding, and physical and emotional exertion. ${ }^{[2]}$ These sudden-onset headaches tend to be bilateral and posterior in location, with a severity that peaks within seconds. ${ }^{[2]}$ In addition, they tend to be short-lived, lasting one to three hours on average, with a milder baseline headache typically persisting between episodes. ${ }^{[2]}$ Other neurologic sequelae, including migrainous features, focal neurologic deficits, seizures, and transient visual deficits are common, with cerebrovascular events possible in severe cases. ${ }^{[2]}$ One study comparing eleven cases found that only sixty-four percent of patients reported thunderclap headaches, while twenty-seven percent had seizures

*Correspondence: Alyssa L. Arena; Email: aarena@wakehealth.edu; Address: Wake Forest School of Medicine, North Carolina, United States. 
and thirty-six percent had motor paralysis. ${ }^{[1]}$ Some cases can also present with autonomic dysfunction, including hypertension in up to half of patients. ${ }^{[1,4]}$ The only consistent objective finding is the diagnostic diffuse vasoconstriction seen on cerebral angiogram. ${ }^{[2]}$ Occasionally, nonspecific cerebrospinal fluid abnormalities are noted. ${ }^{[3]}$ One six-week postpartum patient was determined to have concurrent RCVS and aseptic meningitis, due to her characteristic cerebrospinal fluid findings and pan-negative cultures. ${ }^{[3]}$

Unfortunately the pathophysiology of RCVS is poorly understood. Half of all cases are associated with vasoactive serotonergic and adrenergic drugs, with cannabis as the most common culprit. ${ }^{[2]}$ This suggests a role for sympathetic overactivity. ${ }^{[2]}$ At least one case was associated with the use of oral cyclosporine A and rabbit anti-thymocyte globulin to treat aplastic anemia. ${ }^{[4]}$ Post-partum cases are also common, typically presenting within a week of delivery. ${ }^{[2]}$ This is especially true for women with a history of preeclampsia. ${ }^{[2]}$

There is no established treatment for these severe, refractory headaches. The standard of care relies on elimination of precipitating factors, which led to the resolution of the aforementioned aplastic anemia case. ${ }^{[4]}$ Most patients resolve spontaneously within three months, with no residual findings on angiogram. ${ }^{[2]}$ Nimodipine, verapamil, and magnesium sulfate relieve arterial vasoconstriction, but have had no effect on the course of disease in early studies. ${ }^{[2]}$ Less than one percent of patients die from RCVS, and those that do are often refractory to all treatment. ${ }^{[2]}$ Prognosis is largely dependent on whether the disease is complicated by a cerebrovascular event. ${ }^{[2]}$ Some studies have indicated that up to half of RCVS patients suffer from this complication; however other studies have found no such association. ${ }^{[1]}$

\section{CASE REPORT}

The patient was a twenty eight year-old athletic Caucasian female who was actively breastfeeding at seven months postpartum. She presented with a ten day history of persistent bilateral frontal headaches, with several episodes of sudden escalation to severe pain. Other symptoms included myalgia, nausea, and anorexia, with eventual left-sided hemiparesis, dysarthria, palilalia, and paresthesias. Her past medical and psychiatric history was unremarkable, aside from previous gestational diabetes and mild asthma. She was taking no oral contraceptives or other medications. She denied substance abuse and had a negative urine drug screen. She was borderline tachycardic, likely due to pain, but was otherwise hemodynamically stable and afebrile. Neurological exam confirmed mild left extremity weakness, but was otherwise unremarkable, with negative Kernig and Brudzinski signs. In addition, she had a nonspecific, mildly pruritic, painless left shoulder rash (see Figure 1a). A lumbar puncture revealed cerebrospinal fluid with lymphocyte-predominant pleocytosis, for which she was started on empiric acyclovir, vancomycin, and doxycycline for suspected meningitis. Upon admission to the inpatient medicine unit, her dysarthria and hemiplegia had resolved, although her variable, inconsistent left-sided paresthesias continued throughout her hospital course. Her headaches remained unresponsive to multiple medication trials (see Table 1).

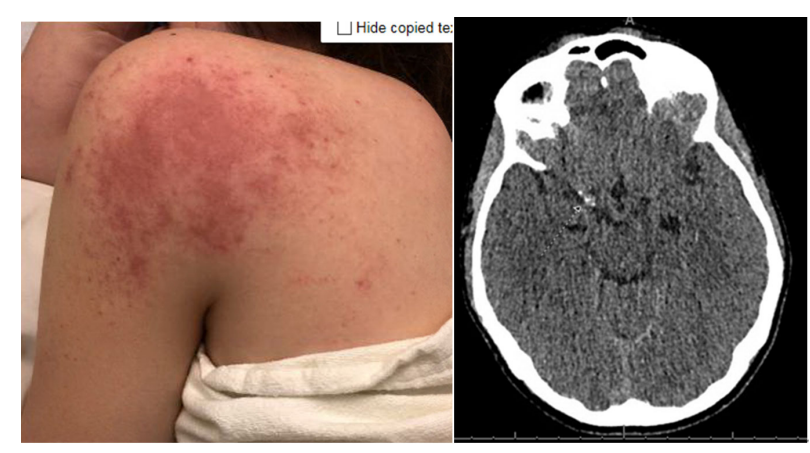

Figure 1. (a) Left: Nonspecific left shoulder rash noted on the patient during admit. (b) Right: Note the possible mild atherosclerosis and ectasia of the distal right internal carotid artery suspected on computed tomography.

A computed tomography revealed possible mild atherosclerosis and ectasia of the distal right internal carotid artery, a finding deemed highly unusual given her age and lack of cardiovascular risk factors (see Figure 1b). Due to her postpartum status, magnetic resonance imaging of her pituitary was performed and was within normal limits.

The patient was a dog groomer who lived with her infant and partner in a rural domicile where ticks had been found in the past. In the midst of these socioeconomic factors and her nonspecific rash, our team was very concerned regarding a vector etiology; however Lyme and West Nile serologies were negative. The rash self-resolved shortly after admission. A summary of her additional negative lab work is provided in Table 2.

Over the course of her two week hospitalization, the patient developed intermittent episodes of delirium, characterized by daytime hypersomnolence and evening insomnia. Moreover, she began to have isolated bradycardic episodes at thirty beats per minute with presyncope. Cardiovascular workup was unremarkable. Her baseline headaches often suddenly worsened overnight, and unfortunately no regimen was successful in relieving these headaches (see Table 1). Attempts to rectify her circadian rhythm, as well as standard delirium precautions, were also unsuccessful. 
Table 1. Headache regimens utilized over the course of our patient's hospital stay and during follow-up period

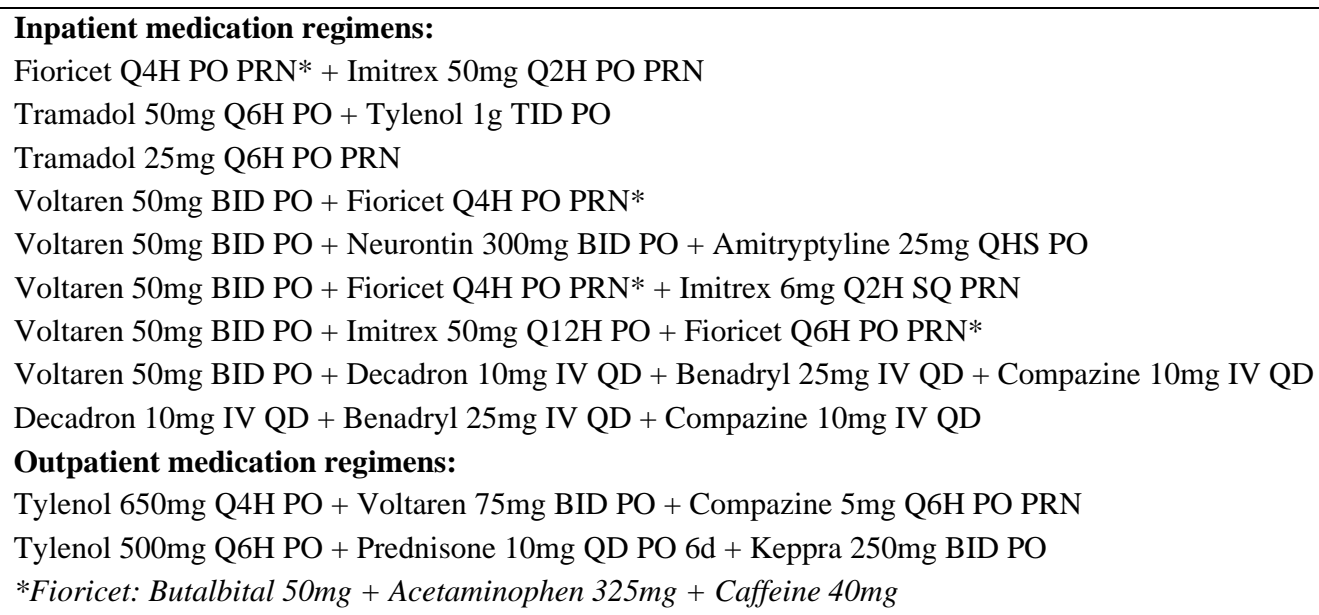

A computed tomography angiogram was performed and revealed right internal carotid artery narrowing, suggesting either reversible cerebral vasoconstriction syndrome or a vasculopathy. Atherosclerosis was determined to be extremely unlikely due to patient age and lack of cardiovascular risk factors. Her imaging also showed mild irregularity of the right M1 segment of the internal carotid artery, and intermittent narrowing and dilation of multiple right M2 branches. There was narrowing of distal middle cerebral artery branches as well.

Following these revelations, a cerebral arteriogram was performed, which confirmed right middle cerebral artery M1 branch tapering toward the artery bifurcation, with fifty to sixty percent vessel stenosis over this short segment (see Figure 2). No other vessel abnormalities that could indicate vasculopathy were noted, and this pattern could not be explained by the possible mild atherosclerosis first noted on her computed tomography head scan, suggesting reversible cerebral vasoconstriction syndrome (RCVS).

Shortly after these results were finalized, sumatriptan, a vasoconstrictor, was discontinued. The patient's sleep improved and her overnight headache exacerbations decreased with no further intervention. She was discharged with prescriptions for Tylenol, Voltaren, and Compazine. Three weeks later the patient reported to follow-up with continued daily headaches that had significantly improved, with no further sudden-onset exacerbations. She maintained some dizziness, nausea, and left arm numbness and tingling, an improvement from her previously more widespread paresthesias. At this time, Tylenol was weaned due to rebound headache concerns, and Prednisone was started to attempt to break the headache cycle. Keppra was started for headache prevention and she was advised to follow-up in six weeks. Unfortunately, the patient did not return for further neurologic follow-up and never received a repeat computed tomography angiogram to determine that status of her cerebral vasculature. However, she was seen three weeks later for an unrelated problem, and had no neurological complaints at that time. She had also discontinued all headache medications.

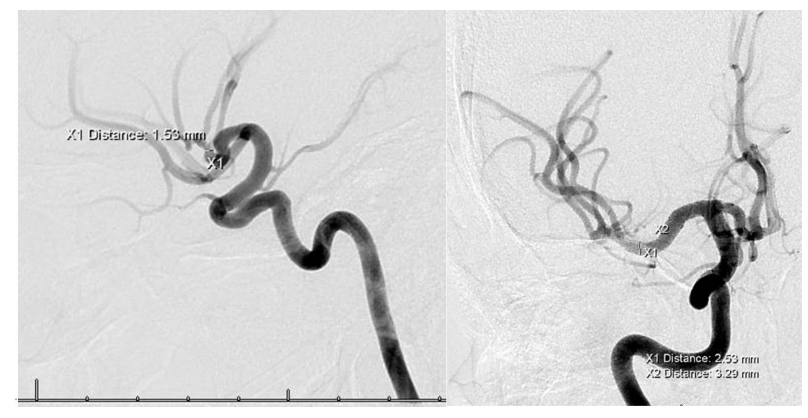

Figure 2. Cerebral arteriogram revealing right MCA M1 smooth tapering from $3.3 \mathrm{~mm}$ diameter to $2.5 \mathrm{~mm}$ prior to MCA bifurcation and $1.3 \mathrm{~mm}$ immediately before bifurcation, with 50\%-60\% vessel stenosis over this short segment

\section{Discussion}

This was a highly unusual case of reversible cerebral vasoconstriction syndrome, given the patient's age and the cooccurrence of suspected aseptic meningitis, confounding her clinical presentation. Accordingly, this is one of the youngest patients documented to have RCVS.

Her subacute, spontaneous resolution further supports the diagnosis of both RCVS and aseptic meningitis, as both conditions would be expected to self-resolve within this timeframe. Although, as covered in our literature review, reversible cerebral vasoconstriction can present with many unusual neurologic and autonomic phenomenon, it seems unlikely that this particular case was not further muddled 
by a second clinical disease. Aseptic meningitis fits her presentation, particularly her episodes of delirium and possible encephalopathy. Her pan-negative serology and cerebrospinal fluid sample with lymphocytic pleocytosis adds further weight to this diagnosis of exclusion. RCVS headaches are notoriously difficult to manage, however superimposed aseptic meningitis likely complicated this treatment regimen further. It is also possible that aseptic meningitis triggered her cerebral vasoconstriction through an unknown method. Complex migraines were also considered, and could also explain her cerebrospinal fluid findings; however migraines were determined to be unlikely given the bilateral, refractory nature of the headaches, without aura. In addition, migraines would not typically be expected to originate and terminate in the span of several weeks, with sudden exacerbations overnight.

Table 2. Infectious and inflammatory serology: All negative unless starred

\begin{tabular}{|c|c|}
\hline Lumbar puncture & $\begin{array}{l}\text { White count 299* (95\% lymphocytes) } \\
\text { Red blood cells } 5 \\
\text { Glucose } 67 \\
\text { Protein } 89\end{array}$ \\
\hline Cultures & $\begin{array}{l}\text { Urine } \\
\text { Blood } \\
\text { Cerebrospinal fluid } \\
\text { Respiratory virus panel }\end{array}$ \\
\hline Meningitis-Encephalitis Panel (cerebrospinal fluid) & $\begin{array}{l}\text { Escherichia Coli } \\
\text { Haemophilus Influenza } \\
\text { Listeria Monocytogenes } \\
\text { Neisseria meningitidis } \\
\text { Streptococcus agalactiae } \\
\text { Streptococcus pneumoniae } \\
\text { Cytomegalovirus } \\
\text { Enterovirus } \\
\text { HSV } 1 \\
\text { HSV } 2 \\
\text { HHV } 6 \\
\text { Human parechovirus } \\
\text { Varicella zoster virus } \\
\text { Cryptococcus neoformans }\end{array}$ \\
\hline Other infectious markers & $\begin{array}{l}\text { Herpes simplex virus } 1 \text { polymerase chain reaction } \\
\text { Herpes simplex virus } 2 \text { polymerase chain reaction } \\
\text { Cryptococcus antigen } \\
\text { Rapid plasma reagin test } \\
\text { Human immunodeficiency virus antibody serology } \\
\text { Lyme antibody } \\
\text { West Nile antibody } \\
\text { Hepatitis A immunoglobulin* (IgM negative) } \\
\text { Hepatitis B surface antibody* (core antibody negative) }\end{array}$ \\
\hline Inflammatory and autoimmune markers & $\begin{array}{l}\text { NMDA antibody } \\
\text { Erythrocyte sedimentation rate } \\
\text { C-reactive protein } \\
\text { Rheumatoid factor } \\
\text { Antinuclear antibody }\end{array}$ \\
\hline
\end{tabular}

Regarding the rash, it was not representative of the classic erythema migrans "bulls-eye" traditionally seen in Lyme disease. Furthermore, the rash would be unlikely to resolve so rapidly if it were due to Borrelia burgdorferi. North Carolina is not a particularly common reservoir for this spirochete, and the patient lacked other classic findings, such as facial palsy and arthritis, that would prompt our team to further explore the possibility of a false-negative test for Lyme. This 
rash may have been an additional manifestation of the likely viral culprit behind her aseptic meningitis. The rash may have also been due to an unrelated allergic skin reaction, given the pruritic nature.

As stated above, most postpartum RCVS cases occur within a few weeks of birth; therefore it was unusual for her to present with this condition at seven months postpartum. Perhaps her postpartum status is unrelated to her RCVS, given this sizable time gap; however no study has clearly delineated the point at which the postpartum risk for RCVS dissipates. To our knowledge, gestational diabetes is not associated with RCVS, unlike preeclampsia. It would be interesting to conduct further studies to determine if the endocrine abnormalities seen in gestational diabetes play any role in RCVS. In her case, an association is unlikely given the span of time since gestation.

Her presentation was typical of RCVS described in the literature, with sudden-onset thunderclap headaches, punctuated by a persistent baseline headache. Interestingly, her headaches were frontal in location, whereas the literature indicates posterior headaches to be more typical of RCVS. She often complained of severe pain from these headaches, however they did seem to wax and wane as the day proceeded, worsening in the evenings and overnight in particular. To our knowledge, there is no established temporal nature of the headaches seen in RCVS. This abnormality in her case could be partially due to likely comorbid aseptic meningitis, with some contribution from delirium and an altered sleepwake cycle. Her focal neurologic deficits and autonomic phenomenon are also in line with a diagnosis of reversible cerebral vasoconstriction syndrome. Her unique bradycardic episodes have not been documented in past literature, which tends to highlight hypertension as a more common autonomic manifestation of RCVS.

Our literature review revealed one other case of co-existing aseptic meningitis and RCVS. Interestingly, this patient presented with aphasia and lateralized findings, mimicking a cerebrovascular event. Our patient had a similar presentation, with left-sided weakness, dysarthria, palilalia, and paresthesias. Although only two such cases have now been reported, it is interesting that both cases of RCVS combined with aseptic meningitis had this stroke-like presentation, and further studies would need to determine if an association exists.

Unfortunately, it seems that no medication regimen was particularly effective for her pain, and that it was largely selfresolving over the course of her hospitalization. This seems to be the typical course of RCVS, emphasized by refractory headaches unresponsive to multiple analgesic trials. Further studies need to be done to determine a better pain regimen for this painful condition. Of note, it may have been helpful to attempt nimodipine, verapamil, or magnesium sulfate to treat her condition, as suggested by literature. It is likely that Imitrex contributed to her headaches through vasoconstriction, and may have slowed her recovery. In fact, the patient seemed to recover somewhat rapidly after the discontinuation of this agent, although this may have also been incidental. We are cautiously optimistic that this patient continued to improve, given her lack of compliance with follow-up and self-discontinuation of her headache regimen. Still, it would have been valuable to repeat the computed tomography angiogram in this patient, to ensure that the vasoconstriction noted in her inpatient scan was indeed fully reversible.

Table 3. Major learning points

- $\quad$ This may be the first documented case of late postpartum RCVS in the context of aseptic meningitis

- $\quad$ This is only the second documented case of RCVS in the context of aseptic meningitis

- $\quad$ RCVS should be suspected in any patient who presents with thunderclap headaches and no subarachnoid hemorrhage on computed tomography head

- $\quad$ RCVS alone may present with cerebrospinal fluid abnormalities, however it is also important to rule out other co-existing disease possibilities, such as aseptic meningitis

- $\quad$ Nonspecific neurological complaints, including stroke-like symptoms, are seen in up to half of RCVS cases

- $\quad$ Autonomic phenomenon in RCVS can include bradycardic and presyncopal episodes

- It is possible that co-existing RCVS and aseptic meningitis is more likely to initially present similarly as a cerebrovascular accident

- $\quad$ A cerebral arteriogram is the gold-standard for RCVS diagnosis

- $\quad$ RCVS headaches are very difficult to treat and it seems the only reliable treatment is identification of and removal of exacerbating and triggering factors

- $\quad$ RCVS patients tend to have a good prognosis with no neurologic sequelae, unless they suffer a cerebrovascular accident during the course of disease 
Table 4. Major points of interest for further reversible cerebral vasoconstriction syndrome research
1 . What is the association between aseptic meningitis and RCVS?
- Is there any specific association with the prognosis of RCVS after recovery?
2. Is there any association between neurologic sequelae seen in RCVS and the identified trigger?
3. At what point does postpartum status stop being a risk factor for RCVS?
4. What is the role of gestational diabetes in RCVS, and how long does this association last postpartum?
- Why do some patients present with postpartum RCVS far later than others?
5. What other autonomic phenomenon are possible with RCVS?
6. Is there an effective medication regimen for RCVS?
- Are nimodipine, verapamil, and/or magnesium sulfate effective in the treatment of RCVS?
7. What other factors affect the prognosis of RCVS?

Her lack of cerebrovascular incident over the course of disease, and apparently in the following few weeks, is also a positive prognostic indicator. It is possible that aseptic meningitis is a more benign precipitator of RCVS, and is less likely to lead to cerebrovascular accident, despite possibly presenting similar to one initially, but further studies must also be done in this area.

\section{Conclusion}

Our unique case of co-existing aseptic meningitis and RCVS is important to document in the literature in order to shed some light on this unusual disease. Her age and latepostpartum status further add to the complexity and distinction of this case. We have provided a list of major learning points form this case in Table 3 .

We believe this case to be the first documentation of late postpartum RCVS in the context of aseptic meningitis. We hope that this report has helped to determine the future trajectory for RCVS research, with a particular emphasis on treatment (see Table 4).

\section{ACKNOWLEDGeMENTS}

We thank the institution of Wake Forest Baptist Health for the great opportunity to care for such interesting, intellectually stimulating patients.

\section{Conflicts of InTERest Disclosure}

The authors have declared no conflicts of interest.

\section{REFERENCES}

[1] Takemaru M. Reversible Cerebral Vasoconstriction Syndrome: A Clinical Study of 11 Cases. Rinsho Shinkeigaku. 2018 Jun 27; 58(6): 377-384. https://doi.org/10.5692/clinicalneurol $. \mathrm{cn}-001143$

[2] Boulos MI, Wortsman J, Swartz RH. Meningitis and Reversible Cerebral Vasoconstriction Syndrome During the Postpartum Period. The
Canadian Journal of Neurological Sciences INC. 2015; 42: 341-343. https://doi.org/10.1017/cjn.2015.268

[3] Ducros A. Reversible Cerebral Vasoconstriction Syndrome. Lancet Neural. 2012; 11: 906-17. https ://doi .org/10.1016/S1474-4 422(12) 70135-7

[4] Yamamoto A, Meguri Y, Fukuda A, et al. A case of reversible cerebral vasoconstriction syndrome developing during treatment of adult aplastic anemia. Annals of Hematology. 2018; 98(2): 511-513. 